\title{
Automated postural responses are modified in a functional manner by instruction
}

\author{
Vivian Weerdesteyn · Andrew C. Laing • \\ Stephen N. Robinovitch
}

Received: 27 April 2007 / Accepted: 18 December 2007 / Published online: 9 January 2008

(C) The Author(s) 2008

\begin{abstract}
The restoration of upright balance after a perturbation relies on highly automated and, to a large extent, stereotyped postural responses. Although these responses occur before voluntary control comes into play, previous research has shown that they can be functionally modulated on the basis of cognitive set (experience, advanced warning, instruction, etc.). It is still unknown, however, how the central nervous system deals with situations in which the postural response is not necessarily helpful in the execution of a task. In the present study, the effects of instruction on automated postural responses in neck, trunk, shoulder, and leg muscles were investigated when people were either instructed to recover balance after being released from an inclined standing posture [balance recovery (BR) trials], or not to recover at all and fall onto a safety mattress in the most comfortable way [fall (F) trials], in both backward and leftward directions. Participants were highly successful in following the instructions, consistently exhibiting stepping responses for balance recovery in BR trials, and sup-
\end{abstract}

\footnotetext{
V. Weerdesteyn $(\square)$

Department of Rehabilitation,

Radboud University Nijmegen Medical Centre,

PO Box 9101, 6500 HB Nijmegen, The Netherlands

e-mail: v.weerdesteyn@reval.umcn.nl

V. Weerdesteyn

Sint Maartenskliniek Research,

Development and Education, Nijmegen, The Netherlands

A. C. Laing $\cdot$ S. N. Robinovitch

School of Kinesiology,

Simon Fraser University, Burnaby, BC, Canada

S. N. Robinovitch

School of Engineering Science,

Simon Fraser University, Burnaby, BC, Canada
}

pressing stepping in the $\mathrm{F}$ trials. Yet EMG recordings revealed similar postural responses with onset latencies between 70 and $130 \mathrm{~ms}$ in both BR and F trials, with slightly delayed responses in $\mathrm{F}$ trials. In contrast, very pronounced and early differences were observed between BR and $\mathrm{F}$ trials in response amplitudes, which were generally much higher in BR than in F trials, but with clear differentiation between muscles and perturbation directions. These results indicate that a balance perturbation always elicits a postural response, irrespective of the task demands. However, when a specific balance recovery response is not desired after a perturbation, postural responses can be selectively downregulated and integrated into the motor output in a functional and goal-oriented way.

\section{Introduction}

The restoration of upright balance after a perturbation relies on highly automated and, to a large extent, stereotyped postural responses, involving a complex pattern of activation of upper and lower leg, trunk, shoulder, and neck muscles (Allum et al. 2002; Bloem et al. 2000; Carpenter et al. 2004; McIlroy and Maki 1995; Moore et al. 1988; Thelen et al. 2000; Woollacott et al. 1988). These responses typically occur at onset latencies of $\sim 100 \mathrm{~ms}$. In response to a perturbation, both feet-in-place and stepping strategies can be used to recover balance, with the incidence of stepping responses becoming larger as the perturbation magnitude increases (Hsiao and Robinovitch, 1998; McIlroy and Maki 1993). These responses are triggered and modulated on the basis of sensory information, with lower extremity and trunk proprioception as well as vestibular inputs as possible sources (Allum and Honegger 1998; Bloem et al. 2000; Do et al. 1988). 
Although the onsets of automated postural responses occur before voluntary (cognitive) control comes into play and the characteristics of the responses are distinctly different from those of voluntary movements (Nashner and Cordo 1981), previous studies have shown that higher brain levels, presumably involving the cerebral cortex, can modulate postural responses by changing the activity of the pathways that are involved in their generation (for review see Jacobs and Horak 2007). In 1976, Nashner was the first to report that experience causes functional adaptations in response amplitudes following an unexpected change of support surface motion. Subsequent studies have shown that response amplitudes depend on the predictability of perturbation magnitudes (Beckley et al. 1991; Horak et al. 1989), restrictions on the balance recovery strategy (feet-in-place vs. stepping; Burleigh and Horak 1996; Burleigh et al. 1994; McIlroy and Maki 1993), and the task of holding an object (Bateni et al. 2004; Marsden et al. 1981). In general, these factors do not cause corresponding changes in onset latencies and activation sequences. On the other hand, both response amplitudes and onset latencies may be affected by advanced warning of the perturbation (Mawston et al. 2007; McChesney et al. 1996) and increased postural anxiety (Carpenter et al. 2004). Hence, the literature indicates that changes in initial contexts generally affect response amplitudes, preserve activation sequences, and have limited influence on onset latencies.

Overall, this pattern of results suggests that the sensory information as generated by the induced perturbation to upright balance inevitably launches a postural response directed toward recovering or maintaining an upright stance, that can be scaled by cognitive set (by changing the gain), but not fully suppressed. However, in all of these previous studies the final goal was the same in every condition, namely balance recovery. It has been postulated (Nashner and McCollum 1985) that in order to reduce the degrees of freedom, the CNS composes complex postural responses from a combination of stereotyped synergies. When a synergy is used to recover balance in response to a perturbation, selective cancellation of single muscle activation within the synergy might not be possible. This raises the question of whether postural responses are also immutable when they are not necessarily helpful in the execution of a task. Would it be possible to turn off these (highly automated) postural responses when functionally undesired, or can they be integrated into the motor output in a meaningful way? The answer could provide important knowledge of the functional organization of the central nervous system in such conflicts. In order to obtain insight into this issue, an experiment would be needed to study postural responses to identical mechanical perturbations, but with distinct task demands.
Instruction-related modulation of long-latency stretch reflexes in response to identical single-joint mechanical perturbations has been extensively studied by instructing participants either to resist or not to resist the perturbation (e.g. Hammond 1956; Rothwell et al. 1980; Gottlieb and Agarwal 1980, Capaday et al. 1994). These studies demonstrated that response amplitudes of the stretched muscles (except for the flexor pollicis longus) were heavily modulated as a result of the instruction, characterized by divergence between instructions (almost) immediately after response onset. Whether such instruction-related modulation also applies to automated postural responses is not known.

In the present study, the effects of instruction on automated postural responses in neck, trunk, shoulder, and leg muscles were investigated when people were either instructed to recover balance after being released from an inclined standing posture, or not to recover at all and fall onto a safety mattress in the most comfortable way. It was hypothesized that instruction would have a profound influence on the whole-body postural response. Two possible scenarios were anticipated. In the first scenario, the instruction of not recovering balance would result in completely different muscle onset latencies, activation sequences, and amplitudes, indicative of a separate motor program (i.e. a set of muscle commands that are structured before a movement sequence begins) to prepare for a safe landing and, consequently, a cancellation of the automated postural response. In the second scenario, the instruction would differentially affect response amplitudes, with no or only limited changes in onset latencies or activation patterns. This pattern of results would imply that the automated postural response could not be suppressed, but could be tailored by differential (feedback and/or feedforward) gain settings in order to meet specific task constraints or demands.

\section{Methods}

Participants

A total of 10 healthy young adults [ 3 women, 7 men, mean age $28.3 \pm 4.3$ years (range 22-37), height $1.74 \pm 0.13 \mathrm{~m}$, weight $68 \pm 13 \mathrm{~kg}$ ] participated in this study. They all provided written informed consent to participate, and the study was approved by the Simon Fraser University Office of Research Ethics.

\section{Procedure}

The participants stood barefoot on a wooden block (length $\times$ width $\times$ height: $60 \times 38 \times 30 \mathrm{~cm}$ ) located flush 
with a gymnasium mattress (length $\times$ width $\times$ height: $480 \times 240 \times 30 \mathrm{~cm})$. A tether was attached at one end to an electromagnetic brake (Warner Electric model PB500, South Beloit, IL) and at the other end to a chest harness worn by the participant (Fig. 1a). The participants placed their feet at a fixed position on the wooden block and the length of the tether was adjusted such that it supported the participant in a $\sim 15^{\circ}$ backward or leftward-inclined position (by means of visual comparison of the lean angle with a reference line). For backward trials, the tether was attached to the front of the harness, whereas for leftward trials, it was attached to the right side of the harness. Postural perturbations were induced by sudden release of the tether (90\% decay time in tether force $=15 \mathrm{~ms})$.

Prior to each trial, the participant was instructed to either recover balance [balance recovery (BR) trials] or to avoid balance recovery attempts and to focus on landing safely [fall (F) trials] after the perturbation. The participants were instructed to lean into the tether and maintain their hips and knees extended, arms flexed, and hands resting on their chest. Before starting the trial, the experimenter confirmed by visual inspection that the head, trunk, and legs were aligned. After the participants indicated they were "ready", the experimenter started the trial. The participants were verbally notified of this event. After the start of the trial, the tether was released at a random time interval, varying between 1 and $7 \mathrm{~s}$. Release from the $15^{\circ}$ lean angle exceeded the maximum value previously observed where young adults can recover using feet-in-place strategies (Hsiao and Robinovitch 2001). Hence, in order to recover, it was necessary for participants to take at least one step. Participants were not constrained regarding which leg to use for stepping. After release, they were also free to move their arms. In the $\mathrm{F}$ trials, participants were not restricted in their use of safe landing responses (such as breaking the fall with the outstretched hands), except that they were not allowed to rotate around the longitudinal axis.

Series of trials were performed for two perturbation directions (backward and leftward). Each participant first performed the series of backward perturbations, followed by the series of leftward perturbations. Each series consisted of six BR trials and six F trials in random order.

Muscle activities in the left and right sternocleidomastoid (SCL and SCR), anterior deltoid (DAL and DAR), posterior deltoid (DPL and DPR), rectus abdominis (ABL and ABR), rectus femoris (RFL and RFR), and tibialis anterior (TAL and TAR) were measured through surface electromyography (EMG; Bagnoli, Delsys Inc., Boston, MA). We considered collecting data from other muscles likely involved in postural responses (e.g. hip abductors and calf muscles) as well. However, in order to prevent harm to both the participants and equipment, we decided not to place electrodes on potential impact sites. The EMG signals were amplified, band-pass filtered $(20-450 \mathrm{~Hz})$ and sampled at $960 \mathrm{~Hz}$. In addition, the 3D positions of skin surface markers were recorded at $240 \mathrm{~Hz}$ with an 8-camera motion analysis system (Motion Analysis Inc., Santa Rosa, CA). Markers were located at the top of the head, sacrum (L5/S1 junction), and bilaterally at the acromion process, lateral epicondyle of the humerus, distal end of the radius, anterior-superior-iliac spine, greater trochanter, lateral epicondyle of the femur, lateral malleolus, and fifth metatarsal. Furthermore, the tether force was recorded at $960 \mathrm{~Hz}$ from a miniature load cell (Sensotec, model 31). Figure $1 \mathrm{~b}$ shows an example of EMG and kinematic data (0-200 ms after tether release) for backward BR and F trials.

\section{Data analysis}

EMG signals were full-wave rectified and low-pass filtered at $25 \mathrm{~Hz}$ (zero-lag, second order Butterworth filter). For each muscle, the mean baseline and standard deviation (SD) in EMG activity over $1 \mathrm{~s}$ prior to tether release were calculated. Tether release was detected as the onset of a sharp decline in tether force. The muscle onset latency was determined by a combination of computer algorithm and visual inspection (to ensure data quality) on a single trial basis. Onset latency for a specific muscle was defined as the time between tether release and the instant the EMG amplitude for that muscle was greater than 4SD's above its baseline value, for at least $30 \mathrm{~ms}$. In addition, for each trial average EMG amplitudes were determined for eight bins following tether release (one bin from 0 to $60 \mathrm{~ms}$, and seven consecutive 20-ms bins from 60 to $200 \mathrm{~ms}$ ). The mean baseline activity of the corresponding condition was subtracted from these values. We only analyzed the first $200 \mathrm{~ms}$ after tether release, because we were particularly interested in whether people would be able to exert cognitive control over the initial part of the postural response, which is presumably highly automated and not generated at the level of the cerebral cortex. As it is well known that reactions at 150-200 ms can certainly be cognitively controlled, EMG signals after $200 \mathrm{~ms}$ were no longer of interest with respect to our primary research question.

We also calculated several kinematic variables to determine whether they would correspond to potential differences in EMG parameters. The instant of step initiation was determined as the first sample after release in which the ankle marker started moving in upward direction. The instant of initial arm abduction was determined as the first lateral movement of the elbow marker. Furthermore, we quantified the initial arm movements by calculating the difference between shoulder flexion and abduction angles at tether release and at $200 \mathrm{~ms}$ post-release. 


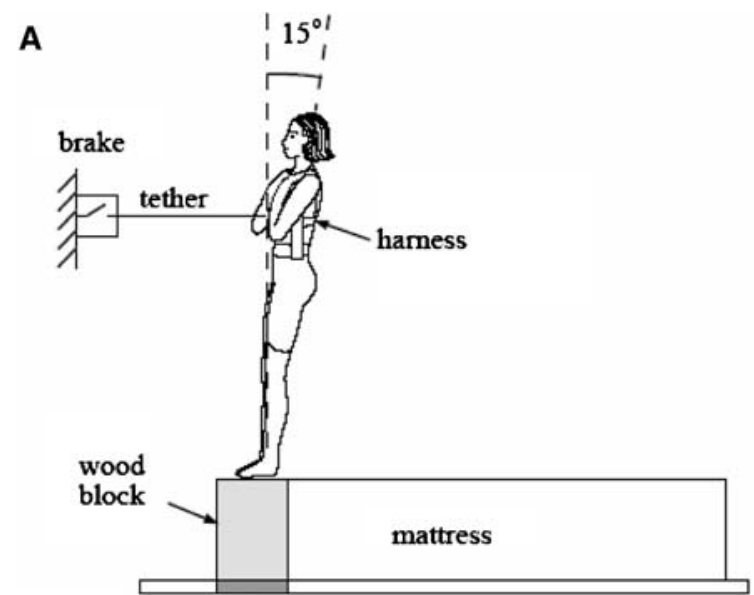

B
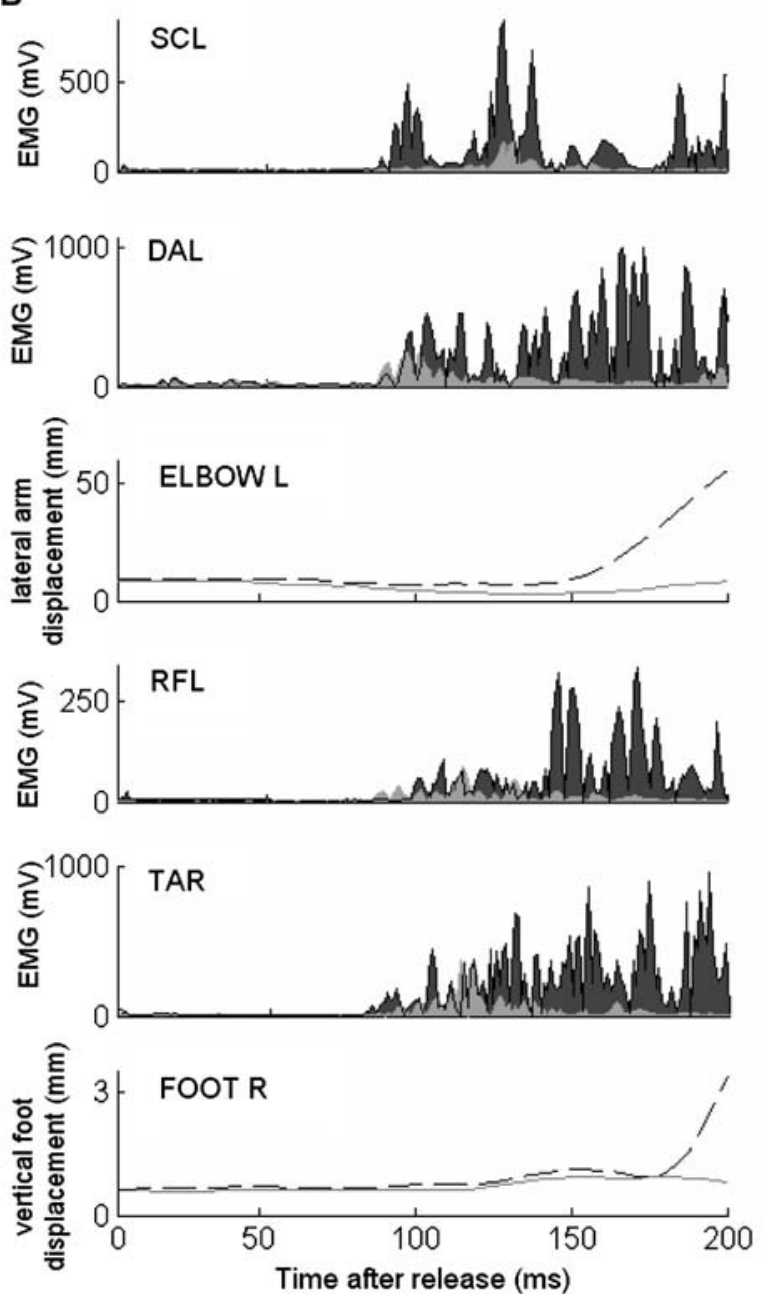

Fig. 1 a Schematic diagram of the experimental setup (backward perturbation position illustrated). Subjects stood supported at an angle of $15^{\circ}$ to the vertical. The tether was released unexpectedly, inducing a balance perturbation. b Raw data from a typical backward balance recovery trial (dark gray area and black, dashed lines) and a fall trial (light grey area and gray, solid lines), showing left sternocleidomastoid EMG (SCL), anterior deltoid EMG $(D A L)$, rectus femoris EMG $(R F L)$, right tibialis anterior $(T A R)$, lateral movement of the left elbow marker, and upward movement of the right foot marker. Tether release is at time $=0 \mathrm{~ms}$
Statistical analysis

Statistical analyses were conducted for data from backward and leftward perturbations separately. Paired $t$ tests were used to identify differences in the instants of tether release after the start of the trial and onsets of arm abduction movements between BR and F trials. Analyses of variance (ANOVA) for repeated measures were used with post hoc paired $t$ tests (with alpha levels adjusted to 0.01 to correct for repeated testing) to detect differences in baseline activity levels and response latencies between instructions. Within-subjects factors were instruction (BR and F), and muscle (12 muscles). ANOVAs for repeated measures were also used for statistical analysis of response amplitudes for each muscle. Within-subjects factors were instruction (BR and $\mathrm{F}$ ), and bins of EMG activity (8 bins following release). When significant Instruction $\times$ Bin interaction effects were present, post hoc reverse Helmert contrasts were used to determine the first bin in which the amplitudes started to deviate between instructions. In addition, ANOVAs for repeated measures were used with post hoc paired $t$ tests (with alpha levels adjusted to 0.01 to correct for repeated testing) to detect the differences in maximum amplitudes and the time of this maximum amplitude between instructions, with instruction (BR and F) and muscle (12 muscles) as within-subjects factors. Finally, paired $t$ tests were used to determine whether changes in arm positions were differentially influenced by instruction. The alpha level was set at 0.05 .

\section{Results}

The average lean angles prior to release were indeed close to the intended $15^{\circ}$, as revealed by analysis of kinematic data from bilateral malleolus and acromion markers. Average lean angles were $15.4 \pm 0.6^{\circ}$ (SE) for backward and $16.9 \pm 0.6^{\circ}$ for leftward trials. There were no significant differences between lean angles in BR and $\mathrm{F}$ trials in both backward and leftward perturbations $(P=0.087$ and $P=0.427$, respectively). Analysis also did not yield significant differences in the instants of tether release relative to the start of the trial (backward: $4.1 \pm 0.24 \mathrm{~s}$ vs. $4.1 \pm 0.27 \mathrm{~s}$ for $\mathrm{BR}$ and $\mathrm{F}$ trials, respectively, $P=0.955$; leftward $4.3 \pm 0.19$ s vs. $4.0 \pm 0.23 \mathrm{~s}, P=0.080$ ).

Of the total $120 \mathrm{BR}$ trials collected in this study, there was only 1 unsuccessful recovery attempt. Furthermore, in only 3 out of $120 \mathrm{~F}$ trials a recovery attempt (step initiation) could be observed. In the backward BR trials, 9 participants stepped back with the right leg first, whereas 1 participant stepped with the left leg first. For this participant, in the statistical analysis of backward perturbations the muscles on the right side were regarded as left, and vice versa. In eight 
participants the stepping foot was the dominant foot (i.e. the foot they use to kick a ball), whereas two participants used their non-dominant foot to step. Foot lift in the backward trials occurred at, on average, $180 \pm 5$ (SE) ms after release. In backward $\mathrm{F}$ trials, most participants landed with near-simultaneous impact to the buttocks and the hands/ forearms, similar to the backward landing configuration reported by Hsiao and Robinovitch (1998). In leftward BR trials, all participants recovered balance by stepping with the left leg first. Left foot lift occurred at $237 \pm 6 \mathrm{~ms}$ after tether release. In leftward $\mathrm{F}$ trials, participants usually landed on the left knee, left hip, and on both left and right hands/forearms.

In response to the perturbation, arm abduction movements were commonly observed in both BR and F trials, but were earlier and more pronounced in $\mathrm{BR}$ than in $\mathrm{F}$ trials. In backward trials, bilateral arm abduction movements occurred at on average $154 \pm 5 \mathrm{~ms}$ after release in BR trials and at $172 \pm 8 \mathrm{~ms}$ in $\mathrm{F}$ trials $(P=0.018)$. In leftward trials, clear abduction movements were usually observed only for the left arm. Movement onsets in BR trials occurred at an average $159 \pm 4 \mathrm{~ms}$ after release and in $\mathrm{F}$ trials at $179 \pm 8 \mathrm{~ms}(P=0.009)$.

Onsets of EMG activity in response to the perturbation could generally be detected in all the muscles within $200 \mathrm{~ms}$. In backward BR trials, neck, trunk and leg muscles showed similar onsets (70-80 ms after tether release), followed by DPL and DPR at 80-90 ms and DAL and DAR at 90-100 ms, on average (Fig. 2). In the backward F trials, the overall mean onset latencies of the 12 muscles measured were delayed by $4.1 \mathrm{~ms}$ ( $\mathrm{SE} 2.4 \mathrm{~ms}$ ) compared to the
BR trials $[F(1,8)=5.601, P=0.045$, Fig. 2]. At the level of the individual muscles, onsets were not significantly different between the instructions (all $P$ values $>0.045$ ). As can be seen from Fig. 2, the overall activation sequence remained similar between instructions.

With respect to muscle activation sequences in leftward BR trials, SCL and SCR were activated first at $75-80 \mathrm{~ms}$ after tether release, followed by ABL, ABR, TAL, TAR, DPL, and DPR at $80-90 \mathrm{~ms}$, DAL and DAR at $90-100 \mathrm{~ms}$, and RFL and RFR at 100-110 ms. Overall, mean onset latencies of the 12 muscles were delayed by $9.3 \mathrm{~ms}$ (SE $4.8 \mathrm{~ms}$ ) in the $\mathrm{F}$ trials compared to the $\mathrm{BR}$ trials $[F(1,8)=20.373, \quad P=0.002, \quad$ Fig. 2$)$. The activation sequence was again generally preserved in the leftward $\mathrm{F}$ trials, with the exception of SCR. The onset of this individual muscle was substantially delayed in F trials compared to $\mathrm{BR}$ trials (mean difference $\pm \mathrm{SE}, 18.6 \pm 5.1 \mathrm{~ms}$, $P=0.007$ ). A less pronounced but significant delay was also observed in DAR (mean difference $\pm \mathrm{SE}$, $9.7 \pm 2.8 \mathrm{~ms}, P=0.006$ ).

With respect to EMG amplitudes, there were no significant differences in baseline activity levels between BR and F trials $[F(1,9)=2.149, P=0.177]$. Hence, the analysis of response amplitudes was not compromised by instructionrelated baseline differences. EMG amplitudes after tether release were generally higher in BR than in F trials and these instruction-related differences could often be detected shortly after onset (mostly within $40 \mathrm{~ms}$ ). For backward trials, significant Instruction $\times$ Bin interactions were found for all muscles [values for $F(7,63)$ ranging from 2.964 to 19.863, all $P$ values <0.010], except ABL, ABR, and RFR
Fig. 2 Average onset latencies $( \pm \mathrm{SE})$ in response to backward and leftward perturbations for left and right sternocleidomastoid (SCL and $S C R$ ), anterior deltoid (DAL and $D A R)$, posterior deltoid ( $D P L$ and $D P R)$, rectus abdominis $(A B L$ and $A B R)$, rectus femoris ( $R F L$ and $R F R$ ), and tibialis anterior $(T A L$ and $T A R)$. Data from balance recovery trials are shown as black diamonds, and data from fall trials are shown as gray squares. $* P<0.01$

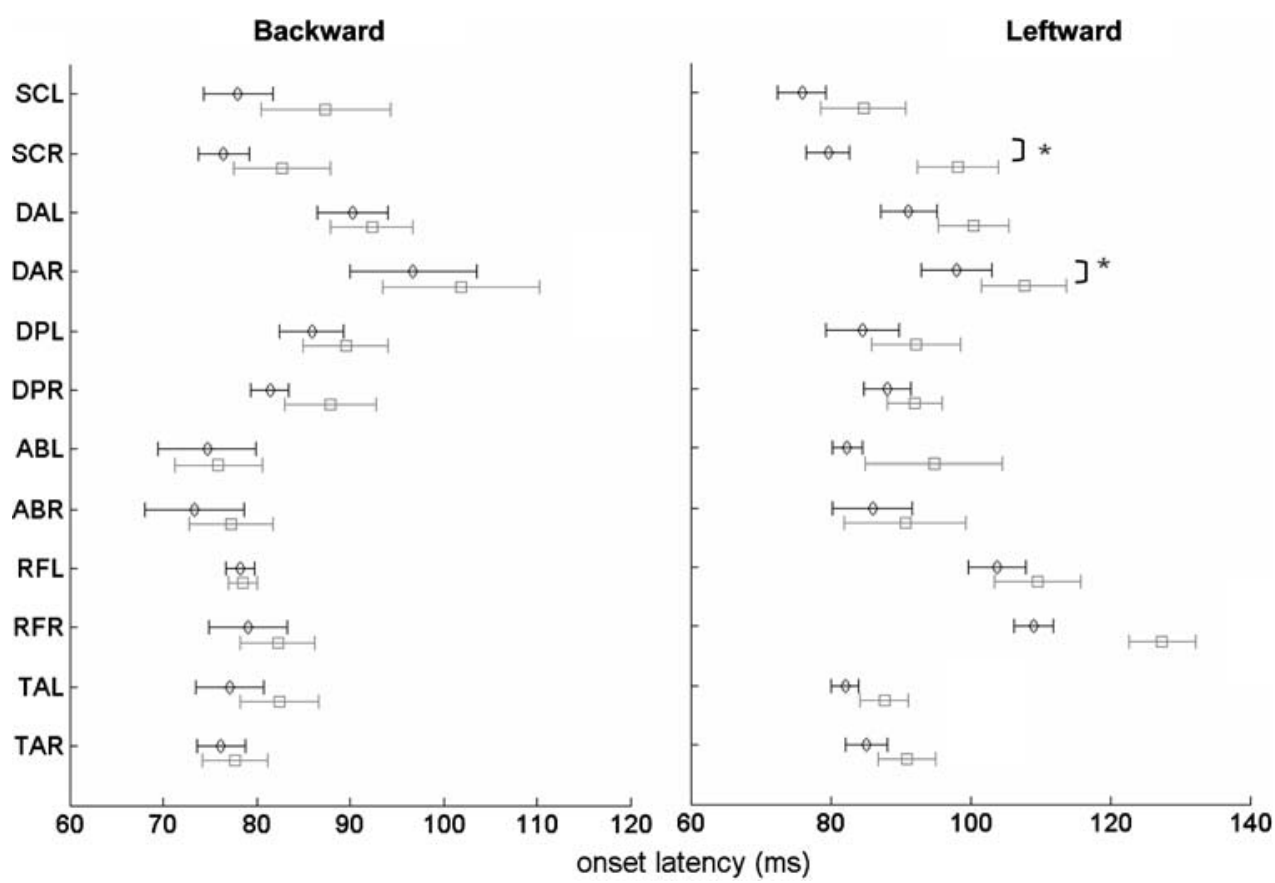


[values for $F(7,63)$ ranging from 0.433 to $1.815, P$ values $>0.100]$. Post hoc contrasts revealed that EMG amplitudes started to increase more steeply in BR than in F trials at 60$80 \mathrm{~ms}$ after release for SCR, at 80-100 ms after release for SCL and TAL, followed by DAL, RFL, and TAR at 100$120 \mathrm{~ms}$, and DAR at $120-140 \mathrm{~ms}$ [values for $F(1,9)$ ranging from 5.946 to $18.575, P$ values between 0.002 and 0.037 ] (Fig. 3).

For leftward trials, analysis yielded significant Instruction $\times$ Bin interactions for all the muscles [values for $F(7,63)$ ranging from 2.424 to 18.294 , all $P$ values $<0.030$ ], except RFL and TAR [values for $F(7,63)$ of 1.317 and $1.198, P$ values of 0.257 and 0.317 , respectively]. Higher EMG amplitudes were observed in BR than in F trials, starting at $80-100 \mathrm{~ms}$ for SCL, SCR, DAL, and ABR, followed by DAR and DPR at 100-120 ms, ABL and RFR at $120-140 \mathrm{~ms}$, DPL at $140-160 \mathrm{~ms}$, and TAL at 180 $200 \mathrm{~ms}$ [values for $F(1,9)$ ranging from 5.157 to 21.745 , $P$ values between 0.001 and 0.049 ] (Fig. 3).

In both backward and leftward perturbations, maximum EMG values (Table 1) were also significantly larger in BR than in $\mathrm{F}$ trials $[F(1,9)=27.430, \quad P=0.001$ and $F(1,9)=33.627, P<0.001]$. The time of maximum EMG was not significantly different between backward $\mathrm{BR}$ and $\mathrm{F}$ trials $[F(1,9)=4.198, P=0.071]$. In leftward trials, analysis yielded a main effect of instruction on the time at which maximum EMG values were reached $[(F(1,9)=8.417$, $P=0.018]$. Post hoc paired $t$ tests revealed that RFR reached peak activity significantly earlier in F than BR tri- als $(P=0.003)$. Continued high RFR activity levels in BR trials probably indicate preparation for the stepping movement of the left leg. Activity levels in the absence of stepping responses ( $F$ trials) started to decrease at $\sim 150 \mathrm{~ms}$.

Hence, many of the muscles recorded showed significantly higher EMG amplitudes in BR than in F trials, with the earliest and most consistent differences between instructions being present in sternocleidomastoid. However, instruction-related differences in rectus abdominis were only present in leftward perturbations. Furthermore, in both perturbation directions, EMG amplitude in rectus femoris of the stance limb was greater in BR than F trials. Finally, instruction had differential effects on shoulder muscle EMG amplitudes for the two perturbation directions. Analysis of shoulder kinematics revealed that the differential effects of instruction on EMG amplitudes also resulted in corresponding changes in shoulder abduction and flexion angles within $200 \mathrm{~ms}$ after tether release (Fig. 4). In backward trials, the deltoids (shoulder abductor muscles) showed higher activity in BR than F trials. This corresponded to increases in shoulder abduction angles in BR trials that were more than twice as large compared to $\mathrm{F}$ trials (left: $10.7^{\circ}$ vs. $4.7^{\circ}$, SE of the difference $2.1^{\circ}, P=0.018$; right: $8.0^{\circ}$ vs. $3.5^{\circ}$, SE of the difference $1.5^{\circ}, P=0.014$, Fig. $4 \mathrm{a}$ ).

In leftward trials, instruction-related differences, with larger amplitudes in $\mathrm{BR}$ than $\mathrm{F}$ trials, were most pronounced in DPR (shoulder abductor and extensor) and DAL (shoulder abductor and flexor). These differences were observed in conjunction with larger shoulder extension
Fig. 3 EMG amplitudes of balance recovery (BR) trials minus the amplitudes in fall (F) trials in response to backward and leftward perturbations. Average differences $( \pm \mathrm{SE})$ are shown as a function of time after tether release for bilateral sternocleidomastoid $(S C)$, anterior deltoid $(D A)$, posterior deltoid $(D P)$, rectus abdominis $(A B)$, rectus femoris $(R F)$, and tibialis anterior $(T A)$. Muscles on the left side of the body are shown as black dashed lines, muscles on the right are shown as gray solid lines. The arrows indicate at which instant EMG amplitudes started to deviate significantly between $\mathrm{BR}$ and $\mathrm{F}$ trials

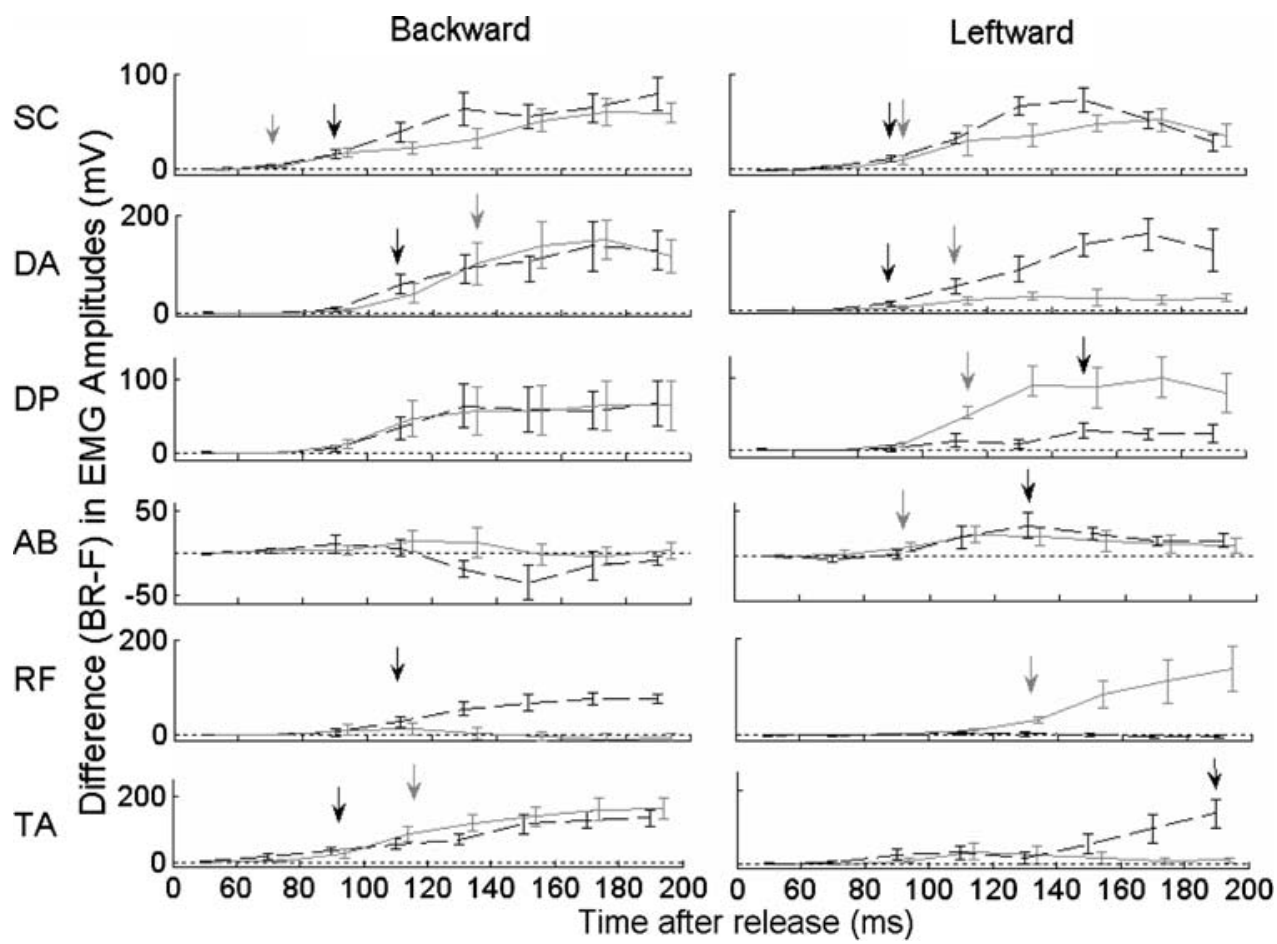


Table 1 Mean \pm SE of maximum EMG amplitude and the instant after release at which the maximum value was reached for balance recovery (BR) and fall (F) trials

\begin{tabular}{|c|c|c|c|c|c|c|c|c|}
\hline & \multicolumn{4}{|l|}{ Backward } & \multicolumn{4}{|l|}{ Leftward } \\
\hline & \multicolumn{2}{|l|}{$\mathrm{BR}$} & \multicolumn{2}{|l|}{$\mathrm{F}$} & \multicolumn{2}{|l|}{ BR } & \multicolumn{2}{|l|}{$\mathrm{F}$} \\
\hline & $\begin{array}{l}\text { Maximum } \\
\text { EMG }(\mathrm{mV})\end{array}$ & $\begin{array}{l}\text { Time of } \\
\max .(\mathrm{ms})\end{array}$ & $\begin{array}{l}\text { Maximum } \\
\text { EMG }(m V)\end{array}$ & $\begin{array}{l}\text { Time of } \\
\max .(\mathrm{ms})\end{array}$ & $\begin{array}{l}\text { Maximum } \\
\text { EMG }(\mathrm{mV})\end{array}$ & $\begin{array}{l}\text { Time of } \\
\max .(\mathrm{ms})\end{array}$ & $\begin{array}{l}\text { Maximum } \\
\text { EMG }(\mathrm{mV})\end{array}$ & $\begin{array}{l}\text { Time of } \\
\text { max. }(\mathrm{ms})\end{array}$ \\
\hline SCL & $217 \pm 21 *$ & $149 \pm 5$ & $124 \pm 21$ & $138 \pm 5$ & $136 \pm 17 *$ & $141 \pm 3$ & $49 \pm 10$ & $140 \pm 11$ \\
\hline SCR & $196 \pm 25 *$ & $155 \pm 2$ & $124 \pm 28$ & $138 \pm 4$ & $112 \pm 23 *$ & $151 \pm 8$ & $32 \pm 9$ & $139 \pm 10$ \\
\hline DAL & $249 \pm 48 *$ & $160 \pm 3$ & $95 \pm 14$ & $144 \pm 7$ & $286 \pm 53 *$ & $163 \pm 4$ & $66 \pm 20$ & $144 \pm 5$ \\
\hline DAR & $239 \pm 50 *$ & $152 \pm 5$ & $51 \pm 9$ & $148 \pm 6$ & $106 \pm 21$ & $148 \pm 7$ & $62 \pm 15$ & $145 \pm 5$ \\
\hline DPL & $161 \pm 66$ & $149 \pm 5$ & $70 \pm 32$ & $139 \pm 4$ & $108 \pm 45$ & $144 \pm 4$ & $56 \pm 26$ & $144 \pm 3$ \\
\hline DPR & $208 \pm 50$ & $141 \pm 5$ & $120 \pm 29$ & $137 \pm 6$ & $211 \pm 51 *$ & $147 \pm 3$ & $71 \pm 21$ & $140 \pm 11$ \\
\hline $\mathrm{ABL}$ & $124 \pm 42$ & $128 \pm 8$ & $139 \pm 40$ & $138 \pm 8$ & $69 \pm 22$ & $140 \pm 8$ & $23 \pm 7$ & $113 \pm 6$ \\
\hline $\mathrm{ABR}$ & $101 \pm 25$ & $131 \pm 6$ & $87 \pm 15$ & $136 \pm 8$ & $57 \pm 17$ & $120 \pm 5$ & $29 \pm 9$ & $112 \pm 7$ \\
\hline RFL & $179 \pm 36$ & $148 \pm 7$ & $119 \pm 20$ & $128 \pm 6$ & $29 \pm 5$ & $144 \pm 6$ & $27 \pm 7$ & $152 \pm 4$ \\
\hline RFR & $93 \pm 19$ & $121 \pm 4$ & $75 \pm 15$ & $134 \pm 5$ & $202 \pm 63 *$ & $173 \pm 3 *$ & $24 \pm 7$ & $156 \pm 4$ \\
\hline TAL & $271 \pm 49 *$ & $162 \pm 6$ & $180 \pm 44$ & $140 \pm 8$ & $270 \pm 48 *$ & $167 \pm 7$ & $179 \pm 37$ & $140 \pm 6$ \\
\hline TAR & $322 \pm 51 *$ & $152 \pm 5$ & $167 \pm 35$ & $131 \pm 8$ & $147 \pm 30$ & $139 \pm 4$ & $122 \pm 26$ & $139 \pm 5$ \\
\hline
\end{tabular}

$* P<0.01$

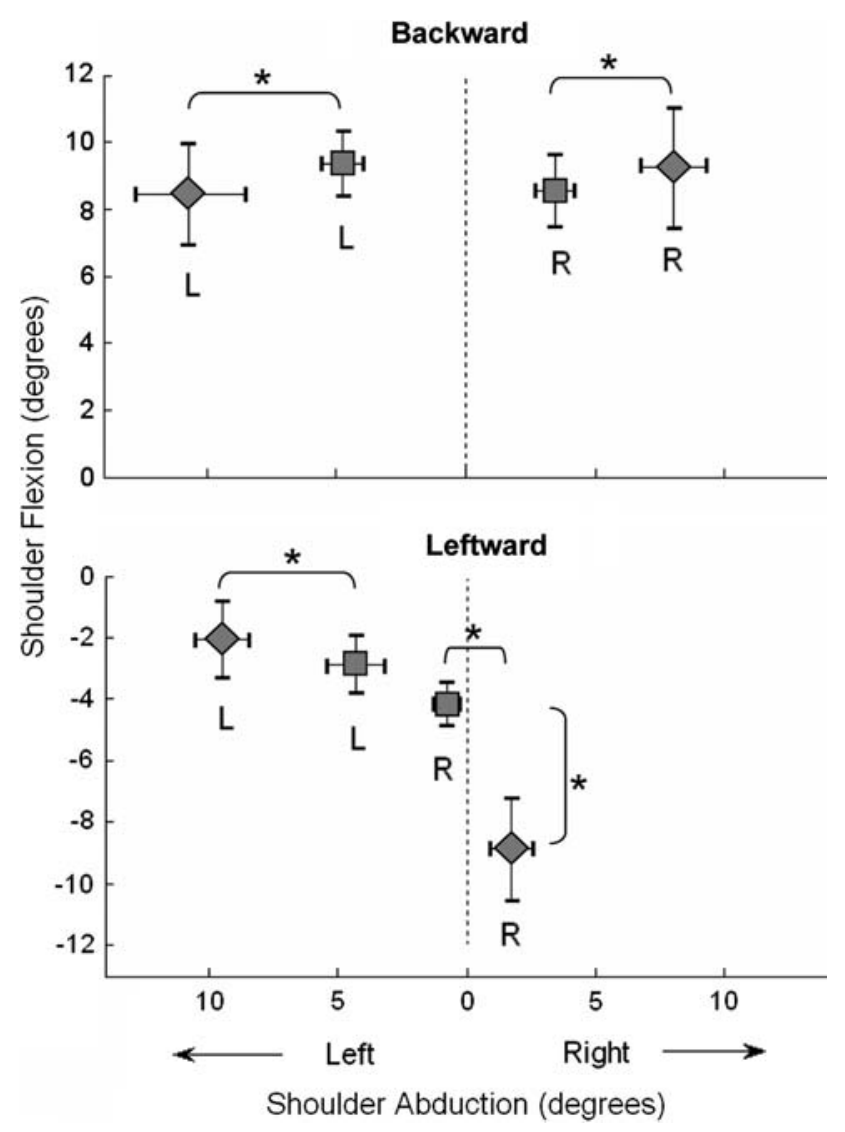

Fig. 4 Average changes in flexion and abduction angles $( \pm \mathrm{SE})$ of the left $(L)$ and right $(R)$ arm between tether release and $200 \mathrm{~ms}$ post-release for balance recovery (diamonds) and fall trials (squares). $* P<0.05$ movements of the right arm in $\mathrm{BR}$ trials $\left(8.9^{\circ}\right.$ vs. $4.2^{\circ}, \mathrm{SE}$ of the difference $\left.1.4^{\circ}, P=0.008\right)$, as well as larger abduction movements of both the left and right arm (left: $9.5^{\circ} \mathrm{vs}$. $4.3^{\circ}, \mathrm{SE}$ of the difference $1.0^{\circ}, P=0.001$; right: $1.7^{\circ} \mathrm{vs}$. $-0.7^{\circ}, \mathrm{SE}$ of the difference $0.7^{\circ}, P=0.007$, Fig. $4 \mathrm{~b}$ ). The right arm was slightly adducted in $\mathrm{F}$ trials, as shown by the average right arm abduction angle being left of the zeroabduction line in Fig. 4b.

\section{Discussion}

The aim of the present study was to investigate the influence of instruction on automated postural responses. Participants were instructed to either recover balance or simply fall onto a gymnasium mat following a sudden postural perturbation. At the behavioral level, participants were highly successful in following these instructions, consistently exhibiting stepping movements for balance recovery in BR trials, and suppressing stepping in the F trials. Yet EMG recordings revealed similar postural responses with onset latencies between $70-130 \mathrm{~ms}$ in both BR and F trials. These onset latencies are in line with those previously reported for forward tether release balance perturbations (Do et al. 1988; Thelen et al. 2000). The presently observed onset latencies are also in the same order of magnitude as the medium-latency responses (80-120 ms) reported for support surface perturbations (Allum et al. 2002; Carpenter et al. 2004), despite the different nature of the perturbations. Only limited instruction-related changes in onset latencies 
were observed, with slightly delayed responses in F trials, in combination with generally preserved activation sequences. In contrast, very pronounced and early differences were observed between $\mathrm{BR}$ and $\mathrm{F}$ trials in response amplitudes, which were generally much higher in BR than in F trials, but with clear differentiation between muscles and perturbation directions. Hence, our results support the hypothesis that instruction influences the early-stage response to a postural perturbation by adjusting multiple gain settings, resulting in targeted scaling across muscles of response amplitudes. However, it does not result in replacement of the motor program.

First, it should be pointed out that the higher response amplitudes in BR compared to F trials could indeed be interpreted as an effect of instruction. Methodological aspects, such as the analysis procedure, cannot explain these amplitude differences. Instruction had no effect on pre-release EMG activity, hence the analysis of response amplitudes was not compromised by instruction-related baseline differences. Furthermore, instruction-related differences in muscle response amplitudes could not be explained by a general time shift of the response, as maximum amplitudes were larger in BR than in F trials and in most muscles, occurred at a similar time after onset of the perturbation.

It may be argued that modulation of response amplitudes could arise from differences in fear of falling between conditions. Carpenter and co-workers (2004) showed that increased levels of postural anxiety resulted in larger response amplitudes after a support surface rotation, in combination with shorter onset latencies in deltoid muscles. In the present study, fear of falling might be expected to be most pronounced in the $\mathrm{F}$ trials. In these trials, however, response amplitudes were reduced and anterior deltoid latencies were delayed. Hence, it seems unlikely that the differences in response amplitudes can be explained by unequal levels of fear of falling between the conditions. This may reflect that, in our experiments, the anxiety associated with the task of recovering balance following sudden release of the tether (although different in nature) may have matched that associated with the task of falling onto the mat, which may of course not be the case during a real-life fall onto a hard surface.

The finding that the two different sets of instructions resulted in modulation of response amplitudes, rather than having major effects on onset latencies and activation sequences, is similar to observations from previous studies (Bateni et al. 2004; Burleigh and Horak 1996; Burleigh et al. 1994; Horak et al. 1989; McIlroy and Maki 1993; Nashner 1976). In these previous studies, a postural response was always required in order to meet the general task demands of maintaining upright balance. Cognitive set resulted in the modulation of response amplitudes of spe- cific muscles within the automated postural response (Bateni et al. 2004; Burleigh and Horak 1996; Burleigh et al. 1994; Horak et al. 1989; McIlroy and Maki 1993; Nashner 1976). The present study provides an important addition to the current body of knowledge by indicating that, even when the occurrence of any postural response may not be desired, it cannot be fully suppressed by instruction, as responses also occurred when people were not supposed to recover, but to fall. The apparently reflex-like generation of postural responses, however, does not seem to interfere with the goal of falling, as participants clearly succeeded following the instruction. This observation provides further insight into the functional organization of the central nervous system. It shows that in balance recovery, we can rely on highly automated responses, but these responses can also be effectively downregulated by the central nervous system when they are not desired.

The present finding that EMG amplitudes were differentially modulated across muscles has been reported previously (Bateni et al. 2004; Burleigh et al. 1994; McIlroy and Maki 1993; Nashner 1976) and it has been suggested that this represents a goal-directed interaction (Burleigh et al. 1994; Ghafouri et al. 2004). The differential effects of instruction, as observed in the present study, are in line with this idea of goal-related changes in response gains.

One result to support the goal-relatedness of EMG modulation was the differential modulation of rectus femoris amplitudes. The higher rectus femoris activation of the stance limb in BR (compared to F) trials can be interpreted as a preparatory action, in order to carry the body weight on one leg to allow stepping with the other. In contrast, in $\mathrm{F}$ trials, no such preparation for weightbearing was needed, which explains the much larger attenuation of the rectus femoris response in the stance than in the stepping leg. The asymmetrical maximum EMG amplitudes of RFL and RFR in the backward F trials, however, seem to indicate that some preparatory activity for stepping may have been present in these trials as well. As previously suggested by Maki et al. (1993), participants may have been unable to suppress the initiation of the preparatory changes in limb loading, but they still may have been able to abort the stepping reaction prior to foot-lift.

Furthermore, the differential modulation of shoulder muscle responses in leftward trials was presumably goalrelated. In leftward trials, the early deviations between instructions of both the shoulder muscle EMG and the corresponding kinematic patterns seemed to be related to positioning the arms to prepare for impact in the F trials, as participants tended to utilize the active response of impacting the ground with the outstretched hands. This is in agreement with the findings as reported by Ghafouri et al. (2004), who observed modulation of early deltoid 
responses when a handrail was available for grasping when balance was perturbed. In their study, arm movements were always directed toward the handrails, irrespective of the direction of the perturbation.

It is insightful to consider the potential mechanisms underlying the slight (and probably not functionally meaningful) delay in onset latencies observed in F trials. This delay cannot be explained by differences in preparation time to the upcoming perturbation, as the instants of tether release were not significantly different between the conditions. This does not exclude the possibility, however, that the delayed responses in $\mathrm{F}$ trials may be related to differences in preparation alertness. The time-critical nature of the BR trials requires a high degree of "readiness" in order to recover balance successfully after the perturbation, whereas this high time pressure is not present in F trials. This difference in time pressure may have induced higher levels of alertness in the BR trials, which is known to facilitate any response (Posner and Boies 1971). Such an effect of alertness on postural responses has been previously reported by McChesney et al. (1996), who found reduced onset latencies when a pre-perturbation warning signal was provided. The present observation that the slight delay in onset latencies was not accompanied by major changes in activation sequences provides additional support for such a generic alertness-related mechanism.

A limitation of the present study was that a relatively small set of muscles was sampled, because EMG electrodes were not positioned on potential impact sites. As such, data could not be collected from the prime movers in response to leftward perturbations (i.e. hip abductors), so it cannot be completely excluded that activation patterns in these perturbations may have been changed as a result of the instruction. Furthermore, forward perturbations (with ventral impact sites in F trials) were not conducted. Although it is conceivable that the presently observed instruction-based modulation of response amplitudes would also apply to forward perturbations, this remains to be determined experimentally.

A second limitation was the predictability of the perturbation direction. This may have influenced the degree to which the reaction could be suppressed, because it allowed participants to preplan their reactions, which is different than most real-life falls or imbalance episodes. It can be expected, however, that these are the most ideal circumstances for suppression of automated postural responses in the $\mathrm{F}$ trials, yet they still occurred in response to the perturbation. It appears that these self-protection mechanisms are so deeply wired in our system that, even under rather optimal conditions, they cannot easily be de-activated.

In conclusion, the present study indicates that automated postural responses occur when balance is perturbed, irrespective of whether people are instructed to recover or not to recover balance. The instruction resulted in differential and probably goal-directed modulation of EMG amplitudes. This suggests that the triggering of postural responses is organized in a reflex-like manner, with supraspinal control primarily contributing to adjust these responses in a functional and goal-oriented way.

Open Access This article is distributed under the terms of the Creative Commons Attribution Noncommercial License which permits any noncommercial use, distribution, and reproduction in any medium, provided the original author(s) and source are credited.

\section{References}

Allum JH, Honegger F (1998) Interactions between vestibular and proprioceptive inputs triggering and modulating human balancecorrecting responses differ across muscles. Exp Brain Res 121:478-494

Allum JH, Carpenter MG, Honegger F, Adkin AL, Bloem BR (2002) Age-dependent variations in the directional sensitivity of balance corrections and compensatory arm movements in man. J Physiol 542:643-663

Bateni H, Zecevic A, McIlroy WE, Maki BE (2004) Resolving conflicts in task demands during balance recovery: does holding an object inhibit compensatory grasping? Exp Brain Res 157(1):49-58

Beckley DJ, Bloem BR, Remler MP, Roos RA, Van Dijk JG (1991) Long latency postural responses are functionally modified by cognitive set. Electroencephalogr Clin Neurophysiol 81:353-358

Bloem BR, Allum JH, Carpenter MG, Honegger F (2000) Is lower leg proprioception essential for triggering human automatic postural responses? Exp Brain Res 130:375-391

Burleigh AL, Horak FB (1996) Influence of instruction, prediction, and afferent sensory information on the postural organization of step initiation. J Neurophysiol 75:1619-1628

Burleigh AL, Horak FB, Malouin F (1994) Modification of postural responses and step initiation: evidence for goal-directed postural interactions. J Neurophysiol 72:2892-2902

Capaday C, Forget R, Milner T (1994) A re-examination of the effects of instruction on the long-latency stretch reflex response of the flexor pollicis longus muscle. Exp Brain Res 100:515-521

Carpenter MG, Frank JS, Adkin AL, Paton A, Allum JH (2004) Influence of postural anxiety on postural reactions to multi-directional surface rotations. J Neurophysiol 92:3255-3265

Do MC, Breniere Y, Bouisset S (1988) Compensatory reactions in forward fall: are they initiated by stretch receptors? Electroencephalogr Clin Neurophysiol 69:448-452

Ghafouri M, McIlroy WE, Maki BE (2004) Initiation of rapid reachand-grasp balance reactions: is a pre-formed visuospatial map used in controlling the initial arm trajectory? Exp Brain Res 155:532-536

Gottlieb GL, Agarwal GC (1980) Response to sudden torques about ankle in man. II. Postmyotatic reactions. J Neurophysiol 43:86-101

Hammond PH (1956) The influence of prior instruction to the subject on an apparently involuntary neuro-muscular response. J Physiol 132:17P-18P

Horak FB, Diener HC, Nashner LM (1989) Influence of central set on human postural responses. J Neurophysiol 62:841-853

Hsiao ET, Robinovitch SN (1998) Common protective movements govern unexpected falls from standing height. J Biomech 31:1-9

Hsiao ET, Robinovitch SN (2001) Elderly subjects' ability to recover balance with a single backward step associates with body configuration at step contact. J Gerontol A Biol Sci Med Sci 56:M42-M47 
Jacobs JV, Horak FB (2007) Cortical control of postural responses. J Neural Transm 114:1339-1348

Maki BE, Whitelaw RS, Mcllroy WE (1993) Does frontal-plane asymmetry in compensatory postural responses represent preparation for stepping? Neurosci Lett 149:87-90

Marsden CD, Merton PA, Morton HB (1981) Human postural responses. Brain 104:513-534

Mawston GA, McNair PJ, Boocock MG (2007) The effects of prior exposure, warning, and initital standing posture on muscular and kinematic responses to sudden loading of a hand-held box. Clin Biomech 22:275-281

McChesney JW, Sveistrup H, Woollacott MH (1996) Influence of auditory precuing on automatic postural responses. Exp Brain Res 108:315-320

McIlroy WE, Maki BE (1993) Changes in early 'automatic' postural responses associated with the prior-planning and execution of a compensatory step. Brain Res 631:203-211

McIlroy WE, Maki BE (1995) Early activation of arm muscles follows external perturbation of upright stance. Neurosci Lett 184:177180

Moore SP, Rushmer DS, Windus SL, Nashner LM (1988) Human automatic postural responses: responses to horizontal perturbations of stance in multiple directions. Exp Brain Res 73:648-658
Nashner LM (1976) Adapting reflexes controlling the human posture. Exp Brain Res 26:59-72

Nashner LM, Cordo PJ (1981) Relation of automatic postural responses and reaction-time voluntary movements of human leg muscles. Exp Brain Res 43:395-405

Nashner LM, McCollum G (1985) The organization of human postural movements: a formal basis and experimental synthesis. Behav Brain Sci 8:135-150

Posner M, Boies S (1971) Components of attention. Psychol Rev 78:391-408

Rothwell JC, Traub MM, Marsden CD (1980) Influence of voluntary intent on the human long-latency stretch reflex. Nature 286:496498

Thelen DG, Muriuki M, James J, Schultz AB, Ashton-Miller JA, Alexander NB (2000) Muscle activities used by young and old adults when stepping to regain balance during a forward fall. J Electromyogr Kinesiol 10:93-101

Woollacott MH, Von Hosten C, Rosblad B (1988) Relation between muscle response onset and body segmental movements during postural perturbations in humans. Exp Brain Res 72:593-604 